\title{
Palladium-Catalyzed Enantioselective Arylation of $\alpha$-Imino Esters
}

Metal-Catalyzed

Asymmetric

Synthesis and

Stereoselective

Reactions

\section{Key words}

palladium

asymmetric arylation

arylglycine derivatives<smiles>CCOC(=O)C=N[Al]</smiles><smiles>CCNC([Al])C(=O)OCC</smiles>

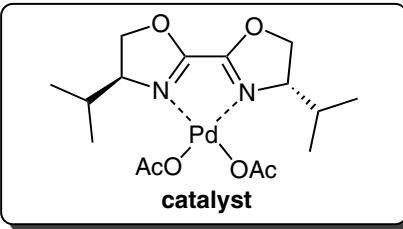

Substrate scope:<smiles>CCOC(=O)[C@H](Nc1ccc(OC)cc1)c1ccccc1</smiles>

$81 \%$ yield, $95 \%$ ee<smiles>CCOC(Nc1ccc(OC)cc1)c1ccccn1</smiles>

no reaction<smiles>CCOC(=O)[C@H](Nc1ccc([N+](=O)[O-])cc1)c1ccccc1</smiles>

$90 \%$ yield, $99 \%$ ee<smiles>CCOC[C@H](Nc1ccc(OC)cc1)c1cccc(C)c1</smiles>

$84 \%$ yield, $96 \%$ ee<smiles>CCOC(Nc1ccc(OC)cc1)c1ccc(Cl)cc1</smiles>

$47 \%$ yield, $94 \%$ ee<smiles>CCO[C@H](Nc1ccc(OC)cc1)c1ccsc1</smiles>

$62 \%$ yield, $93 \%$ ee<smiles>CCO[C@H](Nc1ccc(OC)cc1)c1cccs1</smiles>

$34 \%$ yield, $83 \%$ ee<smiles>CCOC(Nc1ccc(OC)cc1)c1ccoc1</smiles>

$25 \%$ yield, $89 \%$ ee

Proposed transition state:

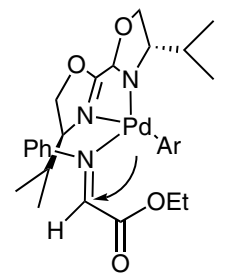

re-face (favored)

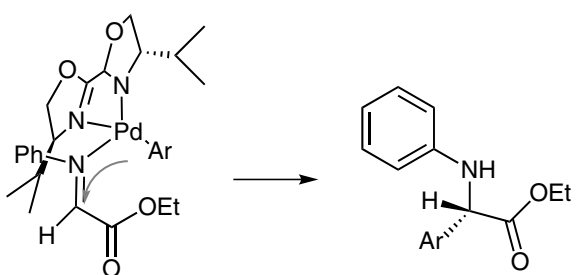

si-face

(disfavored)
Significance: This protocol provides a practical and direct route to chiral arylglycines with high enantioselectivity (up to 99\% ee). These derivatives can be easily converted into optically active $\alpha$-amino acids, which are commonly used as chiral auxiliaries in asymmetric catalysis.
Comment: A palladium(II)-catalyzed asymmetric arylation of $N$-aryl- $\alpha$-imino esters using a chiral BOX ligand was developed. This method is applicable to various aromatic boronic acids. A stereochemical model, consistent with experimental results, suggests a re-face attack of the aryl group onto the $\mathrm{N}$-arylimine carbon.

SYNFACTS Contributors: Hisashi Yamamoto, Lan Luo

Dol: 10.1055/s-0032-1317845; Reg-No.: H16412SF 